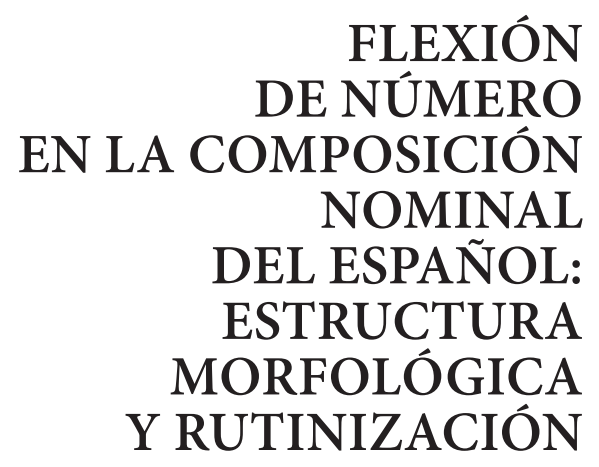


La composición nominal en español es muy heterogénea debido a que existen diversas estructuras con comportamientos distintos. Este comportamiento es evidente en las vacilaciones de los hablantes al marcar el plural de los compuestos: sofás cama o sofacamas; portafolio o portafolios; manos larga o manos largas; etc. Comúnmente, se ha generalizado el comportamiento de la flexión de número como si fuera similar para todos los tipos de compuesto. Así, se considera que la flexión doble se encuentra en compuestos sin amalgama fonológica (actores directores); la flexión interna puede encontrarse en compuestos con núcleo izquierdo, siendo este componente el que recibe las marcas flexivas (hombres rana); finalmente, la flexión marginal aparece en compuestos fusionados, independientemente de la posición del núcleo de la palabra (telarañas). Si bien estas afirmaciones tienen bases empíricas, existen muchas excepciones y contraejemplos (el sacacorchos, las compraventas, los purasangre). En esta investigación partiremos de los preceptos de la gramática cognoscitiva (Langacker, 1999, 2008), y se propondrá que la flexión de número en la composición nominal del español depende de dos factores: primero, la estructura morfológica, que impone una pluralización preferente, y segundo, la rutinización, que provoca un cambio hacia la pluralización marginal conforme el compuesto tiende a integrarse conceptualmente. Con el objeto de contar con datos de habla en uso, se obtuvieron producciones de hablantes nativos de español mediante una encuesta de pluralización de palabras compuestas. Se argumentará que, considerando la interacción de los dos factores mencionados, es posible explicar el comportamiento heterogéneo de la flexión de número en la composición nominal del español.

Palabras clave: flexión de número, composición nominal, lexicalización, morfología, gramática cognoscitiva

In Spanish, compounding is heterogeneous because there are different structures with unique features. This behavior is evident in the speaker's hesitation when marking plural of compounds: sofás cama or sofacamas; portafolio or portafolios; manos larga or manos largas, etc. Typically, scholars have generalized the behavior of number inflection as if it were the same for all types of compounds. Thus, it is taken for granted that double marking number inflection appears in compounds without phonological integration (actores directores); internal marking can be found in left-headed compounds (hombres rana); and finally, marginal number inflection appears in fused compounds, regardless of the position of the word head (telarañas). Although these claims have empirical grounds, there are many exceptions and counterexamples (el sacacorchos, las compraventas, los purasangre). In this paper, I depart from the precepts of cognitive grammar (Langacker, 1999, 2008), and propose that number inflection in nominal composition in Spanish depends on two main factors: first, morphological structure, which imposes a preferential pluralization, and second, entrenchment, which causes a shift toward marginal inflection as the compound word tends to designate unitary concepts. In order to have speech data, productions of plural forms from native speakers of Spanish were elicited. I argue, the interaction of these two factors explains the heterogeneous behavior of number inflection in the nominal composition in Spanish.

KeY WORDS: number inflection, nominal composition, lexicalization, morphology, cognitive grammar 


\section{FLEXIÓN DE NÚMERO EN LA COMPOSICIÓN NOMINAL DEL ESPAÑOL: ESTRUCTURA MORFOLÓGICA Y RUTINIZACIÓN}

Ramón Zacarías Ponce de León Universidad Nacional Autónoma de México

\section{Introducción}

Se ha señalado siempre la complejidad de la flexión de número en las palabras compuestas del español. En su tratado clásico, Alemany (1920: 156) reconoce tres formas distintas de pluralizar. Los compuestos que él llama perfectos, es decir, que se escriben como una sola palabra, solo admiten la pluralización marginal. Para el caso de los compuestos imperfectos, con respecto a los de $\mathrm{N}+\mathrm{de}+\mathrm{N}$ reconoce como única pluralización posible la interna; finalmente afirma 
que para el resto de los imperfectos “....admiten forma plural sólo en uno o en los dos elementos componentes, según éstos hayan llegado o no a fundirse enteramente en uno". Finalmente, afirma que los compuestos $\mathrm{V}+\mathrm{N}$ en los que el segundo elemento aparece en forma plural, carecen de forma propia para marcar su pluralidad.

Las descripciones actuales de la flexión de número en compuestos muestran una situación aún más compleja (Moyna, 2012: 62; NGLE, 2010: 37; Zacarías, 2009: 176; y Bustos, 1986: 202). El primer tipo, de aplicación limitada, es la pluralización doble. Se puede encontrar sobre todo en compuestos de dos sustantivos $(\mathrm{N}+\mathrm{N})$ y es consecuencia de la concatenación sin amalgama fonológica de ambos sustantivos (actores directores, reyes poetas, emisores receptores), donde los dos componentes pueden considerarse parte de un núcleo coordinado. Los compuestos de nombre y adjetivo (N+A) pluralizan también los dos segmentos (pieles rojas, cajeros automáticos, cuentos chinos). Por lo regular, ortográficamente se escriben separados.

El segundo tipo es la pluralización interna (cartas bom$b a$, hombres rana, monos araña). El plural del compuesto se marca en el primer componente, que en estos casos es el núcleo de la estructura. Este tipo de pluralización es muy común porque en español, sobre todo en compuestos $\mathrm{N}+\mathrm{N}$ (perros policía, palabras clave) y $\mathrm{N}+\mathrm{de}+\mathrm{N}$ (estrellas de mar, dientes de ajo), el núcleo es el elemento izquierdo del compuesto y lleva las marcas flexivas de la palabra.

Por último, la pluralización marginal, es decir, aquella que aparece en el margen derecho de la palabra (zarzamoras, coliflores, bocacalles, maniobras, zapapicos), es la más 
común cuando se trata de compuestos fusionados, también conocidos como ortográficos, es decir, aquellos que se escriben como una sola palabra.

Existen, sin embargo, muchos casos problemáticos para la descripción gramatical, como el de la composición de verbo y nombre $(\mathrm{V}+\mathrm{N})$ en la cual el esquema de construcción exige la pluralización del segundo componente para marcar un sentido de iteración o habitualidad (Moyna, 2012: 63; Zacarías, 2009: 51). Por ejemplo, en sacacorchos, el plural corresponde al componente corchos y no al compuesto. De hecho, el compuesto no puede marcar plural: un sacacorchos, dos sacacorchos. Existen casos de lexicalización donde sí puede marcarse el plural del compuesto como en $z a f a$ rrancho: un zafarrancho, dos zafarranchos. Otro caso problemático lo representan los casos donde más de un tipo de pluralización es aceptada. Tal es el caso de la palabra sofá cama donde se detecta vacilación por parte de los hablantes en cuanto a su ortografía y su pluralización. Así, puede encontrarse sofá cama o sofacama. En cuanto a la pluralización, se pueden tener dos opciones: la pluralización interna, sofás cama; y la pluralización marginal, sofacamas. En la sección 2.2, a partir de los resultados de una encuesta sobre formación de plurales, se muestran los casos que provocaron mayor vacilación en los hablantes.

Por un lado, hay que considerar que la estructura morfológica del compuesto impone un tipo de pluralización preferente: la mayoría de los compuestos $\mathrm{V}+\mathrm{N}$ no marcan el plural del compuesto: un abrelatas, dos abrelatas; en los compuestos $\mathrm{N}+\mathrm{de}+\mathrm{N}$ siempre existe pluralización interna: estrellas de mar; en los compuestos $\mathrm{N}+\mathrm{A}$ se marcan ambos 
componentes: cascos azules. Por otro lado, en algunos compuestos parece existir una relación directa entre la pluralización y la integración léxica del compuesto, es decir, el tipo de pluralización puede considerarse como un índice del grado de integración léxica de las unidades compuestas. La pluralización doble correspondería a los compuestos menos integrados (actor director; actores directores), mientras que la pluralización marginal correspondería a los compuestos más integrados y que ortográficamente se escriben como una sola palabra (aguafuerte; aguafuertes).

En esta investigación se buscará demostrar que la flexión de número en las palabras compuestas depende de dos factores; primero, la estructura morfológica del compuesto, la localización del núcleo y las relaciones entre los componentes; segundo, elgrado de integración conceptual que presentala palabra.

\section{Análisis}

Dividimos el análisis en cuatro secciones. En la primera, se explicarán los conceptos de rutinización, integración conceptual y pérdida de analizabilidad que son fundamentales para la propuesta de esta investigación. Después, presentaremos las características de la encuesta que sirvió para la obtención de las producciones de plurales en palabras compuestas y los resultados más relevantes. Posteriormente, describiremos la variación en la flexión de número para cada uno de las seis tipos de compuestos considerados, tomando en cuenta su estructura morfológica, el grado de rutinización y los resultados de la encuesta. En la última sección se 
plantean las principales tendencias de la flexión de número en las palabras compuestas del español.

\subsection{Rutinización de estructuras complejas}

En esta sección se analizará el fenómeno de rutinización, integración conceptual y pérdida de analizabilidad que pueden presentar las palabras compuestas. Se mostrará que en la medida que una palabra se rutiniza sufre un proceso de integración conceptual. Este proceso es paulatino y provoca que la palabra compuesta designe conceptos unitarios aun cuando sea posible reconocer las palabras que intervienen en la composición. Es posible que, en algunos casos, la palabra compuesta se rutinice a tal grado que pierda su analizabilidad y no sea posible reconocer las palabras componentes. En estos casos, la estructura léxica se comportará como una palabra simple tanto a nivel conceptual (concepto unitario), como a nivel morfológico (flexión de género y número). En esta investigación se sostiene que el grado de rutinización y el de integración conceptual pueden influir en la flexión de número del compuesto. A continuación se explicará con mayor detenimiento el proceso de rutinización.

Las estructuras lingüísticas pueden comportarse como rutinas cuando el uso continuo las ha convertido en un hábito. Ciertos eventos, por muy complejos que sean, pueden comportarse como rutinas bien definidas cuando se repiten continuamente. Cada repetición del evento deja tras de sí un "rastro" que hace más fácil posteriores ocurrencias de dicho evento. Cuando una estructura compleja se rutiniza se puede activar sin necesidad de recurrir a sus estructuras 
componentes. Un hablante que utiliza un compuesto rutinizado no necesita ser consciente de sus partes componentes ni de la relación que existe entre ellas. Una expresión de este tipo tiene el estatus de unidad. Siguiendo la nomenclatura de Langacker (1999: 93) representaremos las estructuras que tiene el estatus de unidad como [A]. Una estructura que no tiene estatus de unidad se representa como (A). Para entender mejor lo anterior, vamos a analizar de qué manera interactúan el significado composicional y el significado rutinizado en las estructuras complejas, según Langacker (1999: 112).

Supongamos que tenemos un esquema de construcción que comprende dos estructuras simbólicas componentes [A] y [B] y que ambas forman una estructura simbólica compuesta $([\mathrm{A}][\mathrm{B}])$, que también podemos representar como $([\mathrm{A}][\mathrm{B}])_{\mathrm{C}}$, donde $\mathrm{C}$ representa el valor composicional.

$$
[\mathrm{A}][\mathrm{B}] \rightarrow([\mathrm{A}][\mathrm{B}])_{\mathrm{C}}
$$

$C$ representa el valor composicional que emerge cuando los ítems léxicos $[\mathrm{A}]$ y [B] se integran de acuerdo con las especificaciones del esquema de construcción. La nueva estructura se encierra entre paréntesis redondos porque no tiene el estatus de unidad, por el contrario, su significado es totalmente composicional. Sin embargo, en el uso de las expresiones lingüísticas interviene el contexto. El valor contextual es más específico que el composicional ya que ciertas facetas de la expresión son extracomposicionales. Esto puede representarse:

$$
([\mathrm{A}][\mathrm{B}])_{\mathrm{C}} \rightarrow_{\mathrm{C}}
$$


O bien, de forma abreviada:

$$
\text { (C) } \rightarrow\left(C^{\prime}\right)
$$

(C') es construido en relación con (C); donde (C') representa la manera como es entendida realmente la expresión y es, por lo tanto, su significado contextual.

Finalmente, si la relación de categorización $(\mathrm{C}) \rightarrow\left(\mathrm{C}^{\prime}\right)$ tiene relevancia cognoscitiva, es decir, que el significado composicional figura como parte del significado contextual de la expresión, la relación puede permanecer aunque la expresión, debido a la rutinización, se convierta en una unidad establecida:

$$
[\mathrm{C}] \rightarrow\left[\mathrm{C}^{\prime}\right]
$$

En este caso se utilizan paréntesis cuadrados para indicar que las expresiones, tanto el significado composicional C como el contextual C', tienen el estatus de unidad. Como es difícil distinguir tajantemente entre compuestos con significado puramente composicional y compuestos con significado contextual, lo más apropiado es hablar de un continuo para representar la distancia entre estos dos tipos de significado:

Significado

Significado contextual composicional

$[\mathrm{C}] \rightarrow\left[\mathrm{C}^{\prime}\right]$

sacamuelas sacacorchos

Fig. 1. Significado composicional vs. Significado contextual 
En la figura, se contrastan los compuestos sacamuelas y sacacorchos. En sacamuelas el significado contextual está más presente y por lo tanto se localiza cerca del extremo izquierdo del continuo. Este compuesto designa el oficio de sacar muelas, sin embargo, en el contexto más común, se puede usar para denominar despectivamente a un dentista. En este caso, hay una discrepancia entre el significado composicional y el significado contextual. Por otro lado, en el caso de sacacorchos la palabra compuesta se sitúa del lado derecho porque su significado es composicional; es el instrumento que se utiliza para sacar corchos de las botellas de vino.

\subsubsection{Pérdida de analizabilidad}

La analizabilidad puede entenderse como la probabilidad de que $[\mathrm{A}]$ y $[\mathrm{B}]$, las estructuras componentes, sean activados junto con $[\mathrm{C}]$, la estructura compuesta, es decir, su grado de activación (Langacker, 1999: 127). La analizabilidad reside en la coactivación, junto con la estructura compuesta, de las estructuras componentes que categorizan y motivan a aquélla. La pérdida de analizabilidad se entiende como el grado en el que $[\mathrm{C}]$ puede ser activado independientemen te de $[A]$ y de $[B]$. Dicho de otra manera, existe pérdida de analizabilidad en la medida en que los hablantes dejan de reconocer la presencia de las estructuras componentes. Como ya vimos, una estructura simbólica nueva puede estar muy cerca de la analizabilidad total porque los hablantes han utilizado las estructuras componentes para construir y categorizar dicha estructura nueva. Un factor de la pérdi- 
da de analizabilidad es la rutinización. El hecho de que el uso constante de la expresión la lleve a la rutinización y por tanto a ser considerada como unidad, no implica necesariamente pérdida de analizabilidad, porque dicha unidad, digamos [C], aún puede ser construida y categorizada por $[A]$ y $[B]$. Sin embargo, ahora [C] tiene la posibilidad de ser activada independientemente de $[\mathrm{A}]$ y $[\mathrm{B}]$. Poco a poco las estructuras componentes pierden realidad referencial hasta que el hablante deja de reconocerlas en la activación de [C] y se conserva sólo el significado rutinizado.

El factor más importante en la pérdida de analizabilidad es la discrepancia entre el significado composicional y el significado rutinizado. En la relación de categorización $[\mathrm{C}] \rightarrow\left[\mathrm{C}^{\prime}\right],[\mathrm{C}]$ es el significado componencial a través del cual llegamos a [C']. Conforme va creciendo la discrepancia entre estos dos significados, [C'] es cada vez menos motivado por [C] hasta llegar al punto en que la relación de categorización no tiene realidad cognoscitiva y [C'] es activado autónomamente.

La analizabilidad es una cuestión de grado, y en cada caso hay que decidir hasta qué punto la relación de categorización $[\mathrm{C}] \rightarrow\left[\mathrm{C}^{\prime}\right]$ tiene realidad cognoscitiva $\mathrm{y}$, por tanto, está presente como parte del significado rutinizado. Si comparamos ahora sacacorchos con papanatas $^{1}$ (ambos, compuestos nominales verbo+nombre) parece claro que en el caso de sacacorchos sí está presente el significado composicional, es decir, podemos reconocer tanto el verbo

${ }^{1}$ papanatas. (De papar y nata). 1. com. coloq. Persona simple y crédula o demasiado cándida y fácil de engañar. DRAE (s.v.). 
sacar como el sustantivo corcho. Pero con respecto a papanatas es sólo el significado rutinizado el que se ha codificado como unidad; los hablantes ya no reconocen el verbo papar ni el sustantivo nata. Entre ambos compuestos existe una diferencia en la analizabilidad de la estructura compuesta. Podemos proponer las siguientes representaciones:

\begin{tabular}{|l|l|}
\hline papanatas & sacacorchos \\
{$\left[\right.$ C'] o bien: $^{\prime}$} & {$[\mathrm{C}] \rightarrow\left[\mathrm{C}^{\prime}\right]$ o bien: } \\
[PAPANATAS $]$ & {$[\mathrm{SACA}][\mathrm{CORCHOS}]_{[\mathrm{C}] \rightarrow\left[\mathrm{C}^{\prime}\right]}$} \\
\hline
\end{tabular}

Sin embargo, es difícil trazar una línea clara entre los compuestos que son transparentes porque su relación de categorización tiene realidad cognoscitiva y aquellos compuestos en los que la relación de categorización no es activada junto con el significado rutinizado. Es más exacto hablar de un continuo de analizabilidad donde la distancia entre el significado composicional (C) y el significado rutinizado (C') es gradual. A continuación se contrastan los compuestos sacacorchos y papanatas de acuerdo con su grado de analizabilidad (fig. 2).

Un compuesto muy rutinizado puede perder analizabilidad, es decir, no hay coactivación de las estructuras componentes junto con la estructura compuesta cuando el compuesto es categorizado cognoscitivamente. Esto significa que

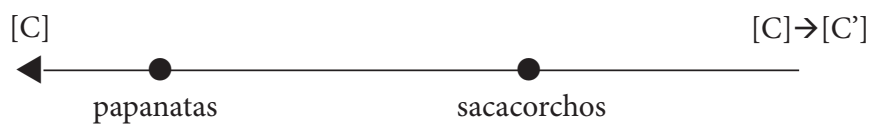

Fig. 2. Pérdida de analizabilidad 
el significado rutinizado discrepa en cierta medida del significado composicional. Cuando esta discrepancia es muy grande, el compuesto presenta una alta pérdida de analizabilidad y hablamos entonces de un compuesto opaco, como en el caso de papanatas.

\subsection{Producciones de plurales de palabras compuestas:}

Encuesta

En esta sección se establecen las características de la encuesta realizada para obtener producciones reales de plurales de compuestos nominales. Asimismo se presentarán los resultados obtenidos y se discutirá su pertinencia para el análisis de esta investigación.

Nuestro objetivo era comprobar y detectar las vacilaciones de los hablantes en la pluralización de palabras compuestas. Con tal objetivo, construimos una encuesta para recabar producciones de palabras compuestas con flexión de número plural. Se elaboró una lista de 63 palabras compuestas (nueva palabras de cada grupo: $\mathrm{V}+\mathrm{N}, \mathrm{N}+\mathrm{A}, \mathrm{A}+\mathrm{N}$, $\mathrm{N}+\mathrm{i}+\mathrm{A}, \mathrm{N}+\mathrm{de}+\mathrm{N}$, y para el caso de $\mathrm{N}+\mathrm{N}$ se incluyeron 18 palabras ya que el núcleo puede estar a la derecha o a la izquierda o ser núcleo coordinado). En la selección de palabras se buscó que hubiera variedad en el tipo de plurales que se podían seleccionar. Para algunas palabras existe sólo una manera de pluralizar y esa respuesta era esperada; sin embargo, se incluyeron palabras para las que era probable que hubiera vacilación por parte de los hablantes: sofá cama, actor director, perro policía, mono araña, rey poeta, casa hogar, tía abuela, chupamirto, portafolio, pelafustán, mano larga, 
cabeza dura, boina verde, cara dura, aguafuerte, purasangre, media naranja, mala leche, malhumor, peliagudo, cabizbajo, cabeciduro, diente de ajo, cabeza de chorlito. Estas palabras presentan distintos grados de rutinización y de integración conceptual, por lo que se esperaba que los hablantes eligieran distintas formas de pluralización.

Una vez diseñada la encuesta, se aplicó a 54 estudiantes de distintos grupos de la Universidad Nacional Autónoma de México (unam) y de la Universidad Autónoma del Estado de México (uafmex). En todos los casos se dictó la lista de 63 palabras con la instrucción de que escribieran la palabra dos veces, en singular y plural. El tiempo de aplicación de la encuesta fue de aproximadamente 15 minutos. En el cuadro 1 se presentan los datos de acuerdo con el número de respuestas y también el porcentaje para cada tipo de plural, para cada una de las 63 palabras compuestas.

Si bien la encuesta no es muy amplia, los resultados obtenidos permiten identificar las vacilaciones de los hablantes en el momento de pluralizar palabras compuestas. Es probable que un estudio más grande arroje más detalles, sin embargo, los datos con los que se cuenta permiten observar tendencias claras, no sólo para cada palabra, sino también por tipo de composición.

En general se nota un alto grado de variación en la formación de plurales, tal como se esperaba. Hay casos como cara dura, en los que se obtuvieron las cuatro posibilidades de pluralización: cara dura (sin marca), caras duras (marcación doble), caras dura (marcación interna) y caraduras (marcación marginal). Es un compuesto de sustantivo y adjetivo, y la pluralización esperada era doble, de acuerdo con 


\begin{tabular}{|c|c|c|c|c|c|}
\hline Tipo & Palabra & $\begin{array}{c}\text { sin } \\
\text { plural }\end{array}$ & $\begin{array}{l}\text { plural } \\
\text { doble }\end{array}$ & $\begin{array}{l}\text { plural } \\
\text { interno }\end{array}$ & $\begin{array}{c}\text { plural } \\
\text { marginal }\end{array}$ \\
\hline \multirow{9}{*}{$\mathrm{V}+\mathrm{N}$} & sacacorchos & $53(98 \%)$ & & & \\
\hline & chupamirto & & & & $54(100 \%)$ \\
\hline & zafarrancho & & & & $54(100 \%)$ \\
\hline & pelafustán & & & & $54(100 \%)$ \\
\hline & portafolio & & & & $54(100 \%)$ \\
\hline & guardapolvo & $6(11 \%)$ & & & $48(89 \%)$ \\
\hline & abrelatas & $54(100 \%)$ & & & \\
\hline & guardabosque & & & & $54(100 \%)$ \\
\hline & papanatas & $52(96 \%)$ & & & \\
\hline \multirow{18}{*}{$\mathrm{N}+\mathrm{N}$} & sofá cama & & & $21(39 \%)$ & $33(61 \%)$ \\
\hline & autopista & & & & $54(100 \%)$ \\
\hline & actor-director & & $48(89 \%)$ & $2(4 \%)$ & $4(7 \%)$ \\
\hline & bocacalle & & $8(15 \%)$ & $2(4 \%)$ & $44(81 \%)$ \\
\hline & compraventa & $4(7 \%)$ & & & $49(91 \%)$ \\
\hline & telaraña & & & & $54(100 \%)$ \\
\hline & zarzamora & & & & $54(100 \%)$ \\
\hline & perro policía & & $12(22 \%)$ & $42(78 \%)$ & \\
\hline & ciruela pasa & & $24(44 \%)$ & $27(50 \%)$ & $3(6 \%)$ \\
\hline & mono araña & & & $42(78 \%)$ & $12(22 \%)$ \\
\hline & palabra clave & & $15(28 \%)$ & $39(72 \%)$ & \\
\hline & coche bomba & & $9(17 \%)$ & $39(72 \%)$ & $6(11 \%)$ \\
\hline & rey poeta & & $42(78 \%)$ & $6(11 \%)$ & $6(11 \%)$ \\
\hline & coliflor & & & & $54(100 \%)$ \\
\hline & radioemisora & $2(4 \%)$ & & & $52(96 \%)$ \\
\hline & casa hogar & & $12(22 \%)$ & $42(78 \%)$ & \\
\hline & tía abuela & & $35(65 \%)$ & $19(35 \%)$ & \\
\hline & autocinema & & & & $53(98 \%)$ \\
\hline \multirow{9}{*}{$\mathrm{N}+\mathrm{A}$} & mano larga & $3(6 \%)$ & $24(44 \%)$ & $3(6 \%)$ & $24(44 \%)$ \\
\hline & \begin{tabular}{|l|} 
cascos azules \\
\end{tabular} & $54(100 \%)$ & & & \\
\hline & cabeza dura & & $21(39 \%)$ & $27(50 \%)$ & $6(11 \%)$ \\
\hline & piel roja & & $45(83 \%)$ & $9(17 \%)$ & \\
\hline & boina verde & & $46(85 \%)$ & $8(15 \%)$ & \\
\hline & cara dura & $9(17 \%)$ & $18(33 \%)$ & $21(39 \%)$ & $6(11 \%)$ \\
\hline & aguafuerte & $8(15 \%)$ & $21(39 \%)$ & $3(6 \%)$ & $22(40 \%)$ \\
\hline & pata chueca & & $46(85 \%)$ & $3(6 \%)$ & $3(6 \%)$ \\
\hline & rabo verde & $9(17 \%)$ & $15(27 \%)$ & $9(17 \%)$ & $21(39 \%)$ \\
\hline \multirow{9}{*}{$\mathrm{A}+\mathrm{N}$} & altavoz & & & & $53(98 \%)$ \\
\hline & purasangre & $45(83 \%)$ & & & $9(17 \%)$ \\
\hline & bajorrelieve & $12(22 \%)$ & $9(17 \%)$ & $2(4 \%)$ & $31(57 \%)$ \\
\hline & Santo Oficio & $12(22 \%)$ & $33(61 \%)$ & & $9(17 \%)$ \\
\hline & media naranja & $6(11 \%)$ & $39(72 \%)$ & $6(11 \%)$ & $3(6 \%)$ \\
\hline & medias tintas & $54(100 \%)$ & & & \\
\hline & mala leche & $19(35 \%)$ & $17(31 \%)$ & $12(22 \%)$ & $5(9 \%)$ \\
\hline & altorrelieve & $12(22 \%)$ & $21(39 \%)$ & $3(6 \%)$ & $18(33 \%)$ \\
\hline & malhumor & $12(22 \%)$ & $24(44 \%)$ & & $18(33 \%)$ \\
\hline
\end{tabular}




\begin{tabular}{|c|c|c|c|c|c|}
\hline \multirow{9}{*}{$\mathrm{N}+\mathrm{i}+\mathrm{A}$} & peliagudo & & & & $54(100 \%)$ \\
\hline & puntiagudo & & & & $54(100 \%)$ \\
\hline & pelirrojo & & & & $54(100 \%)$ \\
\hline & manirroto & & $2(4 \%)$ & & $52(96 \%)$ \\
\hline & manialbo & $6(11 \%)$ & & & $48(89 \%)$ \\
\hline & ojiverde & $11(20 \%)$ & $3(6 \%)$ & & $40(74 \%)$ \\
\hline & cabizbajo & $6(11 \%)$ & & & $48(89 \%)$ \\
\hline & cabeciduro & $10(19 \%)$ & & & $44(81 \%)$ \\
\hline & patizambo & & & & $54(100 \%)$ \\
\hline \multirow{9}{*}{$\mathrm{N}+\mathrm{de}+\mathrm{N}$} & estrella de mar & & & $54(100 \%)$ & \\
\hline & diente de ajo & & & $54(100 \%)$ & \\
\hline & $\begin{array}{l}\text { cabeza de chor- } \\
\text { lito }\end{array}$ & & $9(17 \%)$ & $45(83 \%)$ & \\
\hline & $\begin{array}{l}\text { manos de lum- } \\
\text { bre }\end{array}$ & $54(100 \%)$ & & & \\
\hline & caballito de mar & & & $54(100 \%)$ & \\
\hline & casa de campo & & & $54(100 \%)$ & \\
\hline & ave del paraíso & & & $54(100 \%)$ & \\
\hline & cola de caballo & & & $54(100 \%)$ & \\
\hline & golpe de timón & $4(7 \%)$ & & $52(93 \%)$ & \\
\hline
\end{tabular}

Cuadro 1. Resultados de la encuesta ${ }^{2}$

la estructura morfológica de este tipo de compuestos. Sin embargo, tiene un grado alto de rutinización, lo cual provoca que tienda a la pluralización marginal. Otro caso interesante es el de sofá cama. Es un compuesto de dos sustantivos con núcleo en el componente izquierdo. Debido a esto su pluralización preferencial es la interna (sofás cama). De nueva cuenta, la rutinización provoca una tendencia a la pluralización marginal (sofacamas), que, en este caso, fuela que se presentó con mayor frecuencia.

\footnotetext{
${ }^{2}$ Para algunas palabras los porcentajes no se ajustan al $100 \%$ debido a que no se tomaron en cuenta algunas respuestas de la encuesta, como respuestas nulas o no pertinentes.
} 
Por otro lado, los datos muestran claras tendencias si nos referimos a tipos de composición. Los casos de verbo y sustantivo $(\mathrm{V}+\mathrm{N})$, de sustantivo y adjetivo con vocal de enlace $(\mathrm{N}+\mathrm{i}+\mathrm{A})$ y de dos sustantivos con preposición $(\mathrm{N}+\mathrm{de}+\mathrm{N})$ presentan menos variación, ya que su estructura morfológica impone una preferencia clara por un tipo de flexión de número. Por el contrario, los compuestos de dos sustantivos $(\mathrm{N}+\mathrm{N})$, los de sustantivo y adjetivo $(\mathrm{N}+\mathrm{A})$ y los de adjetivo y sustantivo $(\mathrm{A}+\mathrm{N})$ son los que presentan mayor variación porque su estructura morfológica es menos fija y no impone una preferencia clara por algún tipo de flexión de número. En la sección 2.3 se discutirán estas variaciones con mayor detalle.

En general, podemos afirmar que la encuesta proporcionó datos de primera mano con respecto al tipo de pluralización preferido por los hablantes en distintas circunstancias $\mathrm{y}$ ante distintas estructuras morfológicas. En buena medida los resultados han confirmado nuestras predicciones con respecto a la variación del plural lo que permite afirmar que el tipo de pluralización de la palabra compuesta depende tanto de la estructura morfológica, que es la que condiciona la variabilidad en la posición de la marcación, como de la rutinización, que es el fenómeno que puede considerarse la causa del cambio en la estrategia de los hablantes para marcar la flexión de número y que ésta evolucione hacia una flexión marginal.

\subsection{Descripción de la variación en la flexión de número}

A continuación se describen las características de la flexión de número de los seis tipos de composición nominal que 
hemos tratado en esta investigación y las causas de su variación. Primero se presentan los casos donde la variación es menor: nombre + vocal de enlace + adjetivo $(\mathrm{N}+\mathrm{i}+\mathrm{A})$; verbo + nombre $(\mathrm{V}+\mathrm{N})$; nombre + preposición de + nombre $(\mathrm{N}+\mathrm{de}+\mathrm{N})$; posteriormente trataremos los casos que presentaron mayor variación: nombre + nombre $(\mathrm{N}+\mathrm{N})$; nombre + adjetivo $(\mathrm{N}+\mathrm{A})$ y adjetivo + nombre $(\mathrm{A}+\mathrm{N})$. En este análisis se tomarán en cuenta tanto la estructura del esquema formativo como el grado de analizabilidad de las palabras compuestas.

\subsubsection{Composición $\mathrm{N}+\mathrm{i}+\mathrm{A}$}

Los compuestos de este tipo son llamados perfectos u ortográficos porque presentan una estructura totalmente fusionada que no depende de la rutinización. Son endocéntricos (Moyna, 2012: 132; Zacarías, 2013: 263) y su núcleo es el segundo componente adjetivo. Presentan pluralización marginal: pelirrojo $\rightarrow$ pelirrojos. En los resultados de la encuesta puede observarse que estos compuestos presentan altísimos porcentajes de flexión de número marginal, tal como se esperaba. La estructura del compuesto no varía con el grado de rutinización. Un compuesto antiguo como pelirrojo tiene una estructura similar y una marcación de número plural idéntica a un compuesto novedoso como puede ser ojiver$d e$, de tal manera que no es posible señalar ningún indicio formal de la rutinización. En este caso, notamos en la encuesta que hubo cierta vacilación por parte de los hablantes, ya que una cuarta parte de los encuestados no eligieron la pluralización marginal (ojiverdes). Esto puede deberse a que 
este tipo de compuestos no son productivos en el español de México (Zacarías, 2009b: 33) y, ante la duda, algunos hablantes prefirieron no pluralizar; la mayoría, sin embargo, eligió la flexión marginal. Existen compuestos muy rutinizados como pelirrojo o puntiagudo en los que probablemente los hablantes no pueden reconocer fácilmente las estructuras componentes y por tanto puedan considerarse palabras simples.

\subsubsection{Composición $\mathrm{V}+\mathrm{N}$}

Es un esquema que presenta una flexión de número compleja (Zacarías, 2009: 47). El patrón de formación exige la pluralización del segundo componente para marcar el sentido de habitualidad (un sacacorchos es un instrumento que se utiliza siempre para sacar corchos). Estos compuestos son ortográficos, es decir, están totalmente integrados en una sola palabra y no pueden presentar marcación interna, por lo tanto, la única forma disponible de marcar el plural es la marginal; sin embargo, según el esquema, el segundo elemento se integra en su forma plural. Por ejemplo, en $s a$ cacorchos, el plural corresponde al componente corchos y no al compuesto, el cual queda sin posibilidades de marcar la flexión de número: un sacacorchos, dos sacacorchos. Existen, sin embargo, compuestos cuyo segundo elemento no toma marca de plural como: guardapolvo, quitaesmalte, guardameta, girasol, etc. En estos casos, el lugar de la flexión está desocupado y, por lo tanto, el compuesto sí puede marcar la flexión de número: un guardameta, dos guardametas; un girasol, dos girasoles. En la encuesta, la palabra guardapolvo 
fue pluralizada con flexión marginal en el 89\% de los casos. Los casos que más nos interesan en esta investigación son aquellos en los que, debido a la rutinización del compuesto y a la pérdida de analizabilidad, el segundo componente ya no aparece pluralizado, lo que posibilita la flexión marginal de número del compuesto. Son casos como zafarrancho, pelafustán y portafolio, en los que se puede marcar la pluralidad de la entidad referida por el compuesto: zafarranchos, pelafustanes, portafolios. En la encuesta, para estas tres palabras los hablantes prefirieron la flexión marginal en todas las respuestas. Hay que tomar en cuenta, por otro lado, que existen muchos compuestos $\mathrm{V}+\mathrm{N}$ altamente rutinizados, pero que nunca perdieron la marca de plural, tal es el caso de tragaldabas y papanatas, en los cuales no es posible marcar el plural del compuesto.

\subsubsection{Composición $\mathrm{N}+\mathrm{de}+\mathrm{N}$}

Este tipo de composición puede considerarse como un tipo especial de composición de nombre y adjetivo. El segundo componente modifica al primero, en este caso, a través de la preposición. La flexión de plural se marca siempre en el núcleo izquierdo, es decir, es flexión interna (estrella de mar $\rightarrow$ estrellas de mar). A causa de la preposición interna, estos compuestos no se integran como una sola palabra, por lo que, a pesar de que se puedan encontrar compuestos rutinizados (cola de caballo), la marcación del plural no cambia. Debido a su estructura, principalmente por la preposición interna, estos compuestos, aun cuando se encuentren rutinizados, no presentan variación en su estructura ni cambian 
la pluralización interna. Con los resultados de la encuesta puede corroborarse tal situación, ya que en casi todas las respuestas se eligió la flexión interna. En algunos casos ( $c a-$ beza de chorlito, golpe de timón), se aprecia cierta vacilación, pero los porcentajes no son altos. Resalta el caso de manos de lumbre cuyo primer componente se integra pluralizado en el compuesto. Ante tal situación, no es posible marcar la pluralidad de esta palabra, lo que se corroboró en el total de respuestas de la encuesta.

\subsubsection{Composición $\mathrm{N}+\mathrm{N}$}

Este esquema de composición es el que presenta una mayor variedad en cuanto a las diferencias estructurales y en cuanto a los distintos grados de rutinización que presentan los compuestos de este tipo. En primer lugar, en este tipo de compuestos el núcleo puede ser el primer componente, el segundo, o bien, ambos. Por otro lado, son compuestos donde la fusión de la estructura compuesta depende mucho del grado de rutinización. De esta manera, las posibilidades son muy amplias; para facilidad de análisis, trataremos el grado de analizabilidad de estos compuestos según el lugar que ocupa el núcleo de la construcción.

\subsubsection{Núcleo izquierdo}

En los casos analizados, este esquema presenta dos tipos de pluralización, la nuclear y la marginal. No se presenta la pluralización doble porque el segundo elemento es claramente un modificador del núcleo, es decir, del primer componente, tal como se muestra en los siguientes ejemplos: 
ciruela pasa $\rightarrow$ ciruelas pasa; perro policía $\rightarrow$ perros policía. Cuando estos compuestos se rutinizan pueden presentar flexión marginal: mono araña $\rightarrow$ monoarañas, que fue la respuesta en un $22 \%$ de los casos en la encuesta, o sofá cama $\rightarrow$ sofacamas que apareció en el 61\% de las respuestas. En general, en la encuesta, puede notarse mucha variación en la selección del tipo de flexión, lo que indica que en mayor o menor medida este tipo de compuestos se ve afectado por la rutinización.

\subsubsection{Núcleo derecho}

Este esquema es muy particular, no presenta alta productividad; sin embargo, su estructura está totalmente fusionada debido a que el núcleo es derecho. Sólo presenta un tipo de pluralización, marginal, que además coincide con la pluralización del núcleo. En este caso no hay distinción entre un compuesto totalmente fusionado en el que no pueden distinguirse las estructuras componentes, como zarzamora $\mathrm{y}$ un compuesto no rutinizado, radioemisora, porque ambos pluralizan marginalmente. Los cuatro compuestos de este tipo (zarzamora, radioemisora, autopista y autocinema) incluidos en la encuesta presentan la gran mayoría de respuestas con flexión marginal. En dos respuestas para radioemisora no se marcó el plural y una respuesta para autocinema no fue valida. En pocas palabras, el comportamiento de este tipo de compuesto es muy homogéneo y el tipo de pluralización no es determinado por el grado de rutinización. Podemos decir que el núcleo derecho favorece la integración del compuesto ya que siempre presenta flexión marginal. 


\subsubsection{Núcleo coordinado}

En este caso no hay asimetría entre los componentes; el núcleo es coordinado lo que significa que ambos componentes pueden considerarse nucleares. Raramente se puede encontrar la coordinación de más de un elemento (actor-directorproductor). Pueden pluralizar de dos maneras, doble y marginal. La primera aparece en compuestos muy transparentes como actor director. En la encuesta, el 89\% de las respuestas prefirieron actores directores. La pluralización marginal es muy común en compuestos totalmente fusionados, de acuerdo con la encuesta: coliflores y compraventas, con el $100 \%$ y el $91 \%$ respectivamente. En el caso de tía abuela, el 65\% prefirió pluralización doble, mientras que el 35\% eligió pluralización interna.

\subsubsection{Composición $\mathrm{N}+\mathrm{A}$}

En este tipo de composición, el núcleo siempre es el elemento izquierdo del compuesto, es decir, el sustantivo. En compuestos que no están integrados, la flexión del compuesto se marca en el núcleo, pero en este caso, el adjetivo modificador, elemento derecho del compuesto, concuerda en número y marca también el plural: boinas verdes fue la opción preferida por el $85 \%$ de los encuestados. Como dijimos anteriormente, es un caso de doble marcación por concordancia, no por coordinación. Cuando estos compuestos se rutinizan pueden empezar a marcar el número marginalmente. De la encuesta obtenemos los siguientes valores para la pluralización marginal: aguafuertes, $40 \%$; caraduras, $11 \%$; raboverdes, 39\%; manolargas, $44 \%$. Los datos anteriores nos 
indican que este tipo de composición es particularmente sensible a la rutinización.

\subsubsection{Composición $\mathrm{A}+\mathrm{N}$}

En los compuestos de este tipo, al igual que en el caso anterior, el núcleo es el sustantivo, pero ahora colocado del lado derecho del compuesto. En general son menos productivos que los $\mathrm{N}+\mathrm{A}$. Estas estructuras con el modificador antepuesto, funcionan de manera muy diferente a las anteriores porque su función es más caracterizadora que denominativa. Por eso es difícil encontrarlos en forma plural (media naranja, mala leche, Santo Oficio, malhumor, purasangre). Debido a lo anterior, en la encuesta hubo mucha vacilación en las respuestas ya que los hablantes no están acostumbrados a pluralizar estos conceptos. No sorprende que haya muchas respuestas sin marcación de plural, destacándose purasangre con el $83 \%$ y mala leche con 35\%. Por otro lado, en compuestos con alto grado de rutinización (altavoz, bajorrelieve, altorrelieve) el compuesto es considerado como una palabra simple porque no se evocan las estructuras componentes y presentan frecuentemente pluralización marginal. Los datos de la encuesta son los siguientes: altavoces, $98 \%$; bajorrelieves, $57 \%$; altorrelieves, $33 \%$. En estos casos, el compuesto se usa para denominar entidades individuales y puede establecer la distinción singular-plural. 
2.4. Tendencias generales de la flexión de número en la composición nominal

Después de analizar detalladamente el comportamiento de cada uno de los tipos de composición nominal, con respecto a la flexión de número y a los distintos efectos que produce la rutinización de las palabras compuestas, podemos identificar algunas tendencias generales.

La pluralización menos común es la doble. Se utiliza en compuestos poco integrados que permiten pluralización interna, como los compuestos coordinados (actores-directores). Cuando estos compuestos se rutinizan y se integran tienden a abandonar la pluralización doble a favor de una pluralización marginal como monoarañas, respuesta obtenida en el $22 \%$ de los casos. En el esquema $\mathrm{N}+\mathrm{A}$ encontramos casos de doble pluralización por concordancia (boinas verdes, pieles rojas). De igual manera, al rutinizarse la palabra, la marcación tiende a volverse marginal, como en aguafuertes, que fue la respuesta que se obtuvo en el $40 \%$ de los casos de la encuesta.

La pluralización interna, también conocida como nuclear, aparece cuando se marca la pluralidad en el núcleo y éste es el componente izquierdo. Se utiliza en el esquema $\mathrm{N}+\mathrm{N}$ con núcleo izquierdo (perros policía) y $\mathrm{N}+\mathrm{de}+\mathrm{N}$ (estrellas de mar). En el primer caso, $\mathrm{N}+\mathrm{N}$, el proceso de rutinización provoca que el compuesto tienda a integrarse y por tanto pueda pluralizarse como palabra simple, con flexión marginal, como sofacamas, respuesta obtenida en el $61 \%$ de los casos. En el caso de $\mathrm{N}+\mathrm{de}+\mathrm{N}$, dada su estructura característica, no deja de marcar la flexión de número 
internamente aunque existan procesos de rutinización y pérdida de analizabilidad (cola de caballo).

La pluralización marginal se presenta en los esquemas $\mathrm{N}+\mathrm{i}+\mathrm{A}$ (pelirrojo) y $\mathrm{N}+\mathrm{N}$ (autocinema) con núcleo derecho. Una característica común de estos dos esquemas es que su núcleo es derecho. En estos casos, al identificarse claramente el núcleo, no hay posibilidad de que aparezca doble pluralización; por otro lado, al tener núcleo derecho, tampoco existe la posibilidad de tener pluralización interna, la cual está asociada a compuestos con núcleo izquierdo. De esta manera, la única posibilidad para estos esquemas es la flexión marginal. Este caso, donde coinciden la flexión del núcleo y la flexión del compuesto, puede considerarse el ideal porque facilita la integración de la estructura al no existir flexión interna. En español, sin embargo, no es tan común que el núcleo sea derecho, como es el caso, por ejemplo, de la composición en inglés donde el núcleo siempre es derecho y los compuestos se integran más fácilmente y funcionan, por tanto, como palabras simples. La posición del núcleo es una de las razones por las que la composición en inglés es más productiva que en español. En nuestra encuesta, tanto para el caso de $\mathrm{N}+\mathrm{i}+\mathrm{A}$, como para el caso de las cuatro palabras incluidas de $\mathrm{N}+\mathrm{N}$ con núcleo derecho (autopista, zarzamora, radioemisora y autocinema), se obtuvieron flexiones marginales en casi la totalidad de las respuestas.

Otro caso de posible flexión marginal es el que aparece en ciertos compuestos pertenecientes al esquema $\mathrm{V}+\mathrm{N}$. En este tipo de compuestos se presenta un conflicto entre la flexión de número de la palabra compuesta y la flexión de número del segundo componente. Como ya dijimos, el 
esquema compositivo impone una marca de plural al segundo componente debido al sentido de habitualidad característico de este esquema. El compuesto sacacorchos puede parafrasearse como instrumento para sacar corchos, donde podemos notar que corchos proporciona la idea de una acción repetida muchas veces. Debido a esto, el esquema $\mathrm{V}+\mathrm{N}$ anula la posibilidad de marcar plural de compuesto. Sin embargo, puede existir flexión marginal en aquellos compuestos en los que el segundo componente no aparece en plural. En estos casos, puesto que el lugar de la flexión marginal está libre, el compuesto puede marcar pluralidad. En nuestra encuesta es muy claro que, cuando aparece pluralizado el segundo elemento (sacacorchos, abrelatas, papanatas), la opción de no marcar el plural del compuesto es prácticamente la única respuesta elegida. Por el contrario, en los casos donde el segundo elemento no aparece en plural (chupamirto, zafarrancho, pelafustán, portafolio, guardabosque), el total de respuestas obtenidas fue con pluralización marginal.

\section{Conclusiones}

En esta investigación se analizó la problemática que presenta la flexión de número de las palabras compuestas y su relación con la estructura morfológica del compuesto por un lado, y la integración conceptual de la palabra compleja por el otro. Se estudiaron los conceptos de rutinización y, consecuencia de ésta, la pérdida de analizabilidad de las estructuras compuestas. Estas nociones permiten explicar algunas características relevantes de la composición nominal, 
en particular, la variedad de formas que existen para marcar la flexión de número del compuesto.

En la composición nominal en español existen tres posibilidades de marcación de número: la doble pluralización, la pluralización interna y la pluralización marginal. La marginal es la más natural ya que permite a los hablantes marcar el plural de la palabra compuesta como si fuera una palabra simple. Los compuestos que tienen una estructura totalmente fusionada, como el caso de $\mathrm{N}+\mathrm{i}+\mathrm{A}$, tienen como característica una flexión que no presenta variaciones, siempre es marginal. Además estos compuestos presentan núcleo derecho. Según Haspelmath (2002: 90), el lugar natural para marcar la flexión del compuesto es el extremo derecho de la estructura. Otros compuestos con pluralización marginal, como el esquema $\mathrm{N}+\mathrm{N}$ con núcleo derecho, no son productivos en español. El caso más complejo es el de la composición $\mathrm{V}+\mathrm{N}$, ya que al ser un esquema totalmente fusionado, presenta como única posibilidad la pluralización marginal. Sin embargo, el extremo derecho del compuesto está previamente ocupado por la marca de plural del segundo elemento, que es exigida por el esquema de construcción. Debido a esto, la condición normal de este esquema es no marcar el plural de las entidades referidas.

Otra parte de esta investigación fue el estudio de los procesos de rutinización y pérdida de analizabilidad. En estructuras complejas como las estudiadas en este trabajo, el significado composicional es aportado por las estructuras componentes. Sin embargo, hay una parte del significado de la palabra compuesta que es extracomposicional y depende de los procesos de lexicalización y del contexto de uso de la 
palabra; es el significado contextual del compuesto. Las diferencias que existen entre el significado composicional y el significado contextual se deben al fenómeno de rutinización que sufren los compuestos debido a su uso continuo. Si los compuestos se rutinizan al grado de perder la transparencia de las estructuras componentes, entonces la estructura no es analizable y la palabra ya no es considerada compleja, como en los casos de abrojo y papanatas. Este fenómeno es importante porque, como comprobamos en este análisis, las palabras compuestas pueden presentar distintas formas de pluralización, de acuerdo con la estructura morfológica y el esquema de formación al que pertenecen; pero, al perder analizabilidad, los compuestos dejan de ser estructuras complejas y tienden a comportarse como palabras simples cuya única alternativa es la flexión marginal.

Con el objeto de complementar el análisis y corroborar las tendencias de la flexión de número de los compuestos, se realizó una encuesta sobre pluralización de este tipo de palabras. Si bien este estudio no es amplio, sus resultados nos permitieron comprobar que existe una variación amplia en las formaciones de plurales en la composición nominal. Los resultados del cuadro 1 muestran, por un lado, preferencias de cada esquema compositivo por un tipo de flexión de número y, por otro, que la rutinización de estas estructuras provoca una tendencia hacia la pluralización marginal.

Este análisis nos ha permitido explicar la existencia de una gran variedad de formas de pluralización en la composición nominal, así como la tendencia de todos los esquemas compositivos hacia la pluralización marginal. La rutinización es, según Langacker (1999: 95), un fenómeno básico 
de la cognición humana y, por tanto, no es de extrañar que sea de gran importancia en los procesos de la composición nominal. En esta investigación se ha demostrado que es fundamental tomar en cuenta la rutinizacion y el grado de analizabilidad de las estructuras en el análisis de la morfología del español.

\section{Bibliografía}

Alemany Bolufer, José (1920), Tratado de la formación de palabras en la lengua castellana. La derivación y la composición, Madrid, Librería general de Victoriano Suárez.

Bustos Gisbert, Eugenio (1986), La composición nominal en español, Salamanca, Universidad de Salamanca.

Haspelmath, Martin (2002), Understanding Morphology, Nueva York, Arnold.

LANGaCKer, Ronald (1999), Grammar and Conceptualization, Berlín, Mouton de Gruyter.

- (2008), Cognitive Grammar. A Basic Introduction, Oxford-Nueva York, Oxford University Press.

Moyna, María Irene (2012), Compound Words in Spanish. Theory and history, Ámsterdam / Filadelfia, John Benjamins.

Real Academia Española y Asociación de Academias De la Lengua Española (2010), Nueva gramática de la lengua española. Manual, Madrid, Espasa.

Zacarías Ponce de León, Ramón (2009a), El espacio semántico de la composición nominal, México, Univer- 
sidad Nacional Autónoma de México, tesis de doctorado.

Zacarías Ponce de León, Ramón (2009b), “Posesión inalienable en los compuestos $\mathrm{N}+\mathrm{i}+\mathrm{A}$ del tipo pelirrojo", Lingüística, 21, pp. 31-49.

(2013), “Evolución de la marca de composición 'i’ en las palabras compuestas del tipo bocabierto $\rightarrow$ boquiabierto", en Beatriz Arias, Guadalupe Juárez y Juan Nadal (coords.), Mosaico de estudios coloniales, México, Universidad Nacional Autónoma de México, pp. 261277. 
